\section{The value in computation}

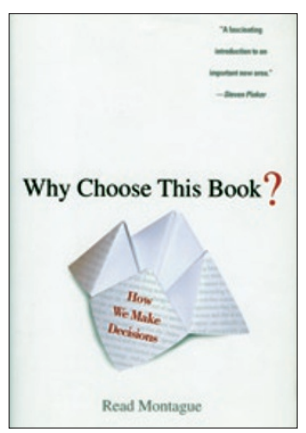

\section{Why Choose This Book? How We Make Decisions}

by Read Montague

Penguin Group (USA), 2006

$352 \mathrm{pp}$, hardback, $\$ 24.95$

ISBN 0525949828

\section{Reviewed by John P O’Doherty}

Since the dawn of the cognitive revolution in the 1950s, researchers have conceived of the brain as an information-processing device, much like a digital computer. Just as terms like buffer, memory store or central processor were being introduced in computer science, so too were similar terms being adopted by cognitive scientists to describe the workings of the mind. Though concepts such as 'working memory', 'long-term memory' and 'attention' were the focus of much interest, aside from the work of a few pioneers this research generally sidestepped the issue of why an individual would bother to store a particular item in memory in the first place or would care enough to direct attention to something.

These days, as anyone taking even a cursory glance at the literature will appreciate, the study of how the brain assigns value to particular stimuli or actions has become de rigueur. In Why Choose This Book?, Read Montague provides an introduction to this new trend in which valuation takes center stage. As Montague points out, animals from bees to human beings must have goals, whether it is foraging for pollen or pursuing a law degree. Among many possible goals, it is imperative to select those behaviors that increase an organism's likelihood of surviving and thus reproducing successfully, by seeking out food and other stimuli that promote survival and avoiding things that hinder that prospect, such as poisons or predators. It stands to reason that the brain should have evolved as a system to facilitate the attainment of such goals. Accordingly, to truly understand brain computation, we need to uncover how the brain comes to assign value to stimuli in the world.

Montague starts out by addressing a topic that seems tangential to the theme of valuation, but does fit into the notion of the brain as a device honed by evolution: efficiency in neural computation. The drive for efficiency is motivated by the need to get as much computation as possible for as little energy as possible. Montague details features of brain architecture that lead to such efficiency, for instance by establishing goals so that some computations are prioritized over others.
The real substance of the book is in chapters four and five: a review of our remarkable progress in understanding the neural mechanisms underlying valuation over the past 15 years. Montague gives prominence to the groundbreaking work of Wolfram Schultz and his colleagues, whose recordings identified a role for dopamine neurons in encoding errors in reward predictions. This finding led Montague, Peter Dayan and Terry Sejnowski to propose a relationship between the functions of these neurons and a class of computational models from a branch of computer science collectively known as reinforcement learning. They suggested that the bursts of activity in these neurons resembled an error signal from an algorithm called temporal difference learning. This class of models can learn flexible predictions of a future reward and can also guide an agent in learning to choose those actions that lead to the greatest probability of attaining that reward. Consequently, these models have been influential in understanding the neural mechanisms underlying action selection for reward, generating insight into the functions of the basal ganglia and frontal cortex along the way.

Montague places this progress in a historical context, from the contributions of Alan Turing in providing some of the theoretical foundations of modern computing, to the work of Richard Sutton and Andrew Barto in developing many reinforcement-learning algorithms. In this regard, more attention could have been given to the contributions of behavioral psychology and animal learning. For instance, psychologists such as Clark Hull and Edward Tolman developed theories of instrumental conditioning that are echoed in modern approaches. Furthermore, temporal difference learning is a direct extension of earlier models from animal learning such as the Rescorla-Wagner rule.

Montague next considers how goals come to be selected in the first place. Many goals relate to basic biological needs, such as obtaining food, water or sex, but others acquire value through association with more basic goals. Although sharks do not go on hunger strikes, in humans a goal can sometimes be very arbitrary and even run counter to basic survival imperatives. For example, some humans take their own lives in a mass cult suicide or as suicide bombers. Montague's intuition is that understanding the basic brain mechanisms of valuation may ultimately help us to understand how such aberrant goal selection can occur-clearly a topical issue at present.

Why Choose This Book? is infused with Montague's infectious enthusiasm for his research, which should inspire students considering this burgeoning field. It constitutes a well-written and accessible introduction to decision-making and should convince anyone remaining to be convinced of the need to consider valuation when trying to unravel the computations of the brain.

Why Choose This Book? was recently issued in paperback with the title "Your Brain is (Almost) Perfect: How We Make Decisions." 